\title{
Autologous Membrane Induced Chondrogenesis (AMIC) for the treatment of acetabular chondral defect
}

\author{
Andrea Fontana \\ Orthopaedic Unit \\ COF Lanzo Hospital, Ramponio Verna (CO), Italy \\ Corresponding author: \\ Andrea Fontana, MD \\ Orthopaedic Unit \\ COF Lanzo Hospital - Department of Orthopaedics 1 \\ 22020 Ramponio Verna (CO), Italy \\ E-mail: dr.andreafontana@libero.it
}

\section{Summary}

Background: Acetabular chondral defect are very frequently associated to FAI. Treatment options are still questionable.

Methods: Between 2008 and 2014, 201 patients over 583 have been arthroscopically treated with the AMIC procedure for grade III and/or IV acetabular chondral lesions. Patients age was between 18 and 50 years; acetabular chondral lesion size was between 2 and $4 \mathrm{~cm}^{2}$; radiological Tönnis degree of osteoarthritis was $\leq 2$.

Results: The mean follow up of the entire group of 201 patients was 5 years (from 8 to 2). Significant improvement, as measured by the mHHS, was observed at 6 months in comparison to preoperative levels $(80.3 \pm 8.3)(p<0.001)$. Continuous improvement with respect to each previous evaluation time point was seen, reaching the highest improvement level at the three year follow-up $(85.5 \pm 7.2)$. The mean $\mathrm{mHHS}$ improvement recorded at the five year follow-up compared with preoperative scores was $39.1 \pm 5.9$.

Conclusions: AMIC is a valid procedure to repair medium-sized chondral defects on the acetabular side of the hip found during treatment of FAI and lead to long-term favourable outcomes.

Level of evidence: IV.

KEY WORDS: hip chondral defects, AMIC (Autologous Membrane Induced Chondrogenesis), FAl (Femoro Acetabular Impingemet), hip arthroscopy.

\section{Introduction}

During the past decade a dramatic change has occurred in the diagnosis and treatment of hip pathologies. While many new pathoanatomic features, such as femoroacetabular impingement (FAI), labral pathologies and developmental dysplasia have been described, hip arthroscopy has become popular as an attractive alternative to the more invasive open surgeries ${ }^{1}$.

There is actually agreement in considering chondral lesions as a consequence of other pathological features such as trauma, osteonecrosis, dysplasia, labral tears, loose bodies, dislocation, previous slipped capital femoral epiphysis and Femoro Acetabular Impingement $(\mathrm{FAI})^{2,3}$. In recent years, $\mathrm{FAl}$ in particular, gained growing attention and has been indicated as a cause of osteoarthritis of the hip ${ }^{4,5}$. According to this theory, the altered morphology of the femur and/or of the acetabulum results in abnormal contact against the joint, thereby leading to stress degeneration of the labrum and cartilage.

Many options for biologic joint reconstruction as an alternative to arthroplasty exist, such as microfractures, osteochondral autograft transplantation, mosaicplasty, autologous chondrocyte implantation, etc ${ }^{6}$. Most of these techniques have a long and successful clinical history ${ }^{7-10}$

In contrast, in the case of hip surgery, excellent results have been reported using the arthroscopic treatment of $\mathrm{FAl}$, removal of symptomatic loose bodies and many other intra and extra articular hip pathologies. Nevertheless, concerns remain about the correct treatment of chondral lesions ${ }^{11,12}$.

This article discusses the innovative option for the treatment of chondral lesions in the hip with the AMIC technique.

\section{Material and methods}

Between 2008 and 2014, 201 patients over 583 treated with hip arthroscopy, underwent an AMIC procedure for the treatment of grade III and/or IV acetabular chondral lesions, according to the Outerbridge classification ${ }^{13}$. All these 201 patients included in this retrospective, non randomized study have been selected according to the following inclusion criteria: chondral lesions were located in the superior area of the acetabulum; they were consequent to $\mathrm{FAI}$; $\mathrm{Pa}$ tients age was between 18 and 50 years; acetabular chondral lesion size was between 2 and $4 \mathrm{~cm}^{2}$; radiological Tönnis degree of osteoarthritis was $\leq 2$. Exclusion criteria were: concomitant presence of femoral head chondral lesion; systemic rheumatoid diseases; dysplasia; femoral neck axial deviations; coxa profun- 
da and/or protrusio acetabuli. For FAl cam-type, arthroscopic femoral head-neck resection arthroplasty was performed to restore the anatomic offset between the femoral head and neck. In case of FAl pincer-type, arthroscopic acetabular rim trimming was performed to reduce the bony overhang and to reshape the acetabulum into its normal contour. An eventual detached labrum was reattached to the superior acetabular rim with suture anchors. Intra operative dynamic tests were performed moving the hip along its full range of motion, checking the absence of any remaining bony impingement.

The AMIC procedure was performed arthroscopically in a single surgical stage ${ }^{14,15}$. The chondral defect was measured with an arthroscopic probe and standard microfracures (MFX) were carried out. Bone marrow bleeding from the holes was verified after removing the fluids from the joint space by continuous aspiration. Destroyed and unstable cartilage was removed using angled curettes or motorised shavers to achieve a wellcontained defect. The Chondro-Gide ${ }^{\circledR}$ matrix, a resorbable bilayer collagen I/III membrane (ChondroGide ${ }^{\circledR}$, Geistlich Pharma AG, Wolhusen, Switzerland), was cut to fit the size and the shape of the lesion and placed on the chondral defect with the porous layer facing the bone surface through an arthroscopic cannula (Figure 1). The post-operative rehabilitation programme started on the first post-op day. Patients began rehabilitation with isotonic and isometric quadriceps and gluteus contractions. Walking was allowed with the aid of two crutches with partial weight-bearing (30\% of body weight) on the operated leg for three weeks. Cycling exercises started from post-operative day two, swimming was allowed after two weeks. At
Table I. Baseline characteristics of the study groups

AMIC $(n=201) p$

- Sex (M/F) 13/18

- FAl-cam (n) 21

- FAl-plncer (n) 16

- FAl-comblned (n) 6

- Preoperative mean age (years) 36.4 \pm 10.3 (18-55)

- Preoperatlve mean defect slze (cm2) $2.9 \pm 0.8$ (2-4)

- Preoperative mean mHHS 44.9 \pm 5.9 (38-60)

four weeks post-op, walking with the aid of one crutch opposite to the treated leg was allowed for seven days, then normal walking thereafter. Impact sport activity could resume at three months post-op and complete return to sport activities was allowed six months after surgery. All patients were assessed preoperatively and at follow-up after 6, 12 months and than yearly, using the modified Harris Hip Score $(\mathrm{mHHS})^{16}$. The $\mathrm{mHHS}$ assesses hip function with a maximum score of 91 . Our results were rated as follows: excellent (81-91), good (71-80), fair (61-70) and poor (less than 60) ${ }^{17}$. The study was approved by the IRB of the Institute and all patients gave their consent to the data collection and publication.

\section{Results}

The mean follow up of the entire group of 201 patients was 5 years (from 8 to 2). The average age was $36.4 \pm 10.3$ years. The mean defect size was

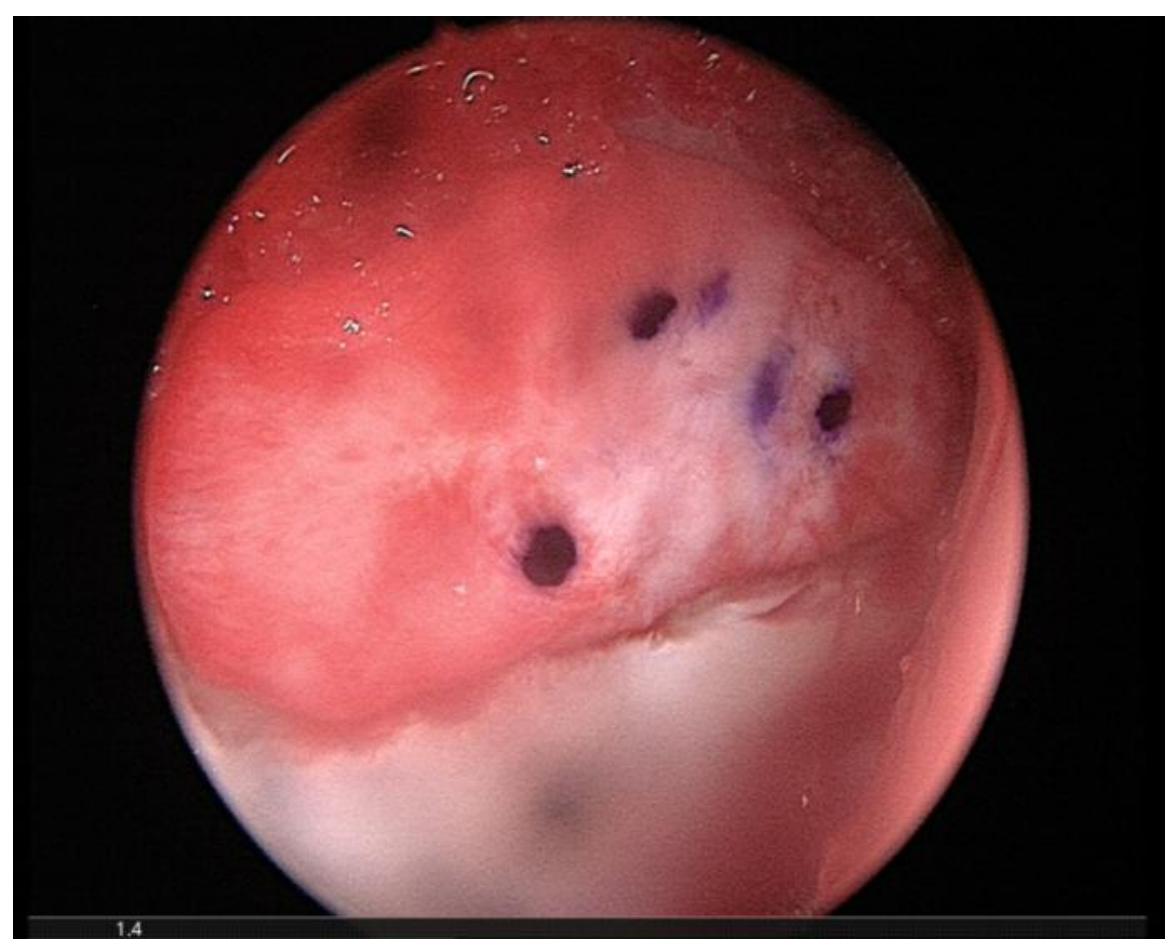

Figure 1. Chondro gide membrane is applied to cover the acetabular chondral defect. The implant has been marked on its smooth surface to allow the correct placement of its rugh face in contact with the subchondral bone. 


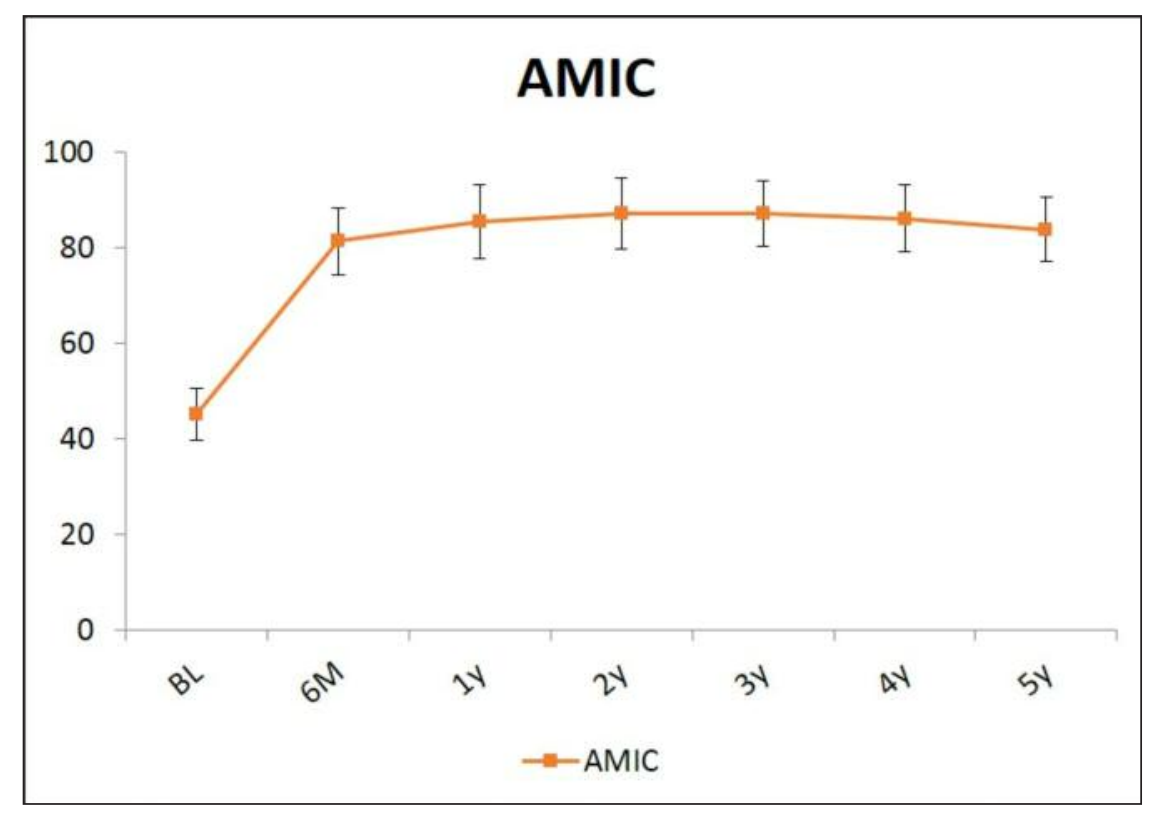

Figure 2. Pre-operative mHHS and up to 5 years after AMIC.

$2.9 \pm 0.8 \mathrm{~cm}^{2}$. Pre-operative $\mathrm{mHHS}$ had a mean score of $44.9 \pm 5.9$. Significant improvement, as measured by the $\mathrm{mHHS}$, was observed at 6 months in comparison to preoperative levels $(80.3 \pm 8.3)(p<0.001)$. Continuous improvement with respect to each previous evaluation time point was seen, reaching the highest improvement level at the three years follow-up $(85.5 \pm 7.2)$. The $\mathrm{mHHS}$ then remained stable over time until the final years follow-up (Figure 2). At each of the $12,24,36,48$ and 60 months time points, the $\mathrm{mHHS}$ was significantly higher in comparison to the 6 month values. The mean $\mathrm{mHHS}$ improvement recorded at the 5 years follow-up compared with preoperative scores was $39.1 \pm 5$.9. No patient had a poor postoperative $\mathrm{mHHS}(>60)$.

No significant complications were reported and no failure resulting in hip arthroplasty was detected in any of these patients during the five year follow-up.

\section{Discussion}

Our study reports on long-term clinical outcomes for repair of $2-4 \mathrm{~cm}^{2}$ chondral lesions with the AMIC technique, assessed by mHHS. Significant improvements were demonstrated up to the 5 years follow-up, demonstrating that AMIC can be reliably extended to $4 \mathrm{~cm}^{2}$ defects. Several arthroscopic techniques have been used to treat full-thickness chondral defects of the hip in the past few decades. However, due to the introduction of hip arthroscopy for the treatment of FAI, published outcome studies on hip cartilage repair are scarce and comprehensive evidence-based treatment guidelines for chondral lesions of the hip remain to be defined. MFx is still currently the first choice treatment for both acetabular and femoral head small chondral defects $\left(\leq 2 \mathrm{~cm}^{2}\right)$ as it is not in- vasive and very rarely has it been associated with major post-operative complications. Satisfactory clinical results after MFx of the hip 2,11,18-20, including athletes ${ }^{9,21}$, have been recently reported; however, the follow-up reported for MFx in the hip did not exceed two years ${ }^{9,19,22}$. Thus, clinical data relating lesion size, treatment choice and evaluation of cartilage repair procedures beyond two years are of critical importance to determine predictable and sustainable therapy for chondral cartilage defects of the hip. Literature on the use of $\mathrm{MACl}$ for the treatment of medium-sized chondral defects in the hip is particularly scarce. The first case report by Akimau et al. ${ }^{23} \mathrm{de}-$ scribed the treatment of an extensive loss of cartilage and osteonecrosis in the hip treated with bone grafting and $\mathrm{MACl}$ using Chondro-Gide ${ }^{\circledR}$. Larger controlled retrospective studies, comparing arthroscopic AMIC with debridement ${ }^{14}$ and with $\mathrm{MFx}^{24}$ for the treatment of grade III/IV acetabular chondral defects larger than $2 \mathrm{~cm}^{2}$, have been recently reported demonstrating very good results at 5 years of follow up in the group treated with the AMIC technique.

The AMIC technique spares donor site morbidity since effective cartilage regeneration can be stimulated in a single surgical intervention without the need for harvesting cells from a second site. AMIC exploits the regenerative potential of mesenchymal progenitor cells deriving from subchondral bone. The collagen type I/III matrix used in AMIC protects the blood clot and supplies the regenerating site with a proper microenvironment supporting cell adhesion, growth and differentiation. Collagen matrices have previously been shown to support chondrogenic differentiation of mesenchymal stem cells ${ }^{25}$ and to maintain chondrocyte phenotype ${ }^{26}$, in particular when the matrix is composed of collagen types I and $\mathrm{III}^{27}$. The AMIC technique is further beneficial because it eliminates 
the need for specialised centres and laboratory support to cultivate cells, in turn reducing total therapy time and overall cost, compared to two-stage procedures such as $\mathrm{MACl}$. Our findings are in line with the results deriving from the treatment of cartilage defects of the knee and talus with the AMIC technique ${ }^{28-31}$. Gille et al. ${ }^{28}$ showed that patients affected by large grade IV chondral lesions experienced significant improvement in terms of five different evaluation scores at 12 months and up to 24 months after the AMIC procedure. Satisfactory outcomes were also reported for osteochondral lesions of the talus treated by AMIC 32,33.

Until now, long-term outcome data for the treatment of chondral defects of the hip using the AMIC treatment were not available. One of the limitations of our study is that it is a retrospective analysis of data collected over years of treatment. Another limitation is that clinical improvement was evaluated based only on the mHHS. Although this test has high validity and reliability ${ }^{16}$, it is most suitable for assessment of functionality in elderly arthritic patients and might not be sensitive enough to assess subtle changes in function in young, otherwise healthy patients. Nevertheless, the improvement in these scores suggests that AMIC provides clinical benefits to patients affected by a chondral lesion consequent to femoral acetabular impingement.

Other parameters like age and weight of the patient and morphology of the hip and soft tissues have not been deeply evaluated in this study. Those factors can influence the the results and should be taken in consideration.

The study was conducted according to international the ethical standards ${ }^{34}$.

\section{Conclusions}

In conclusion, the AMIC technique allowed a marked clinical improvement in patients affected by chondral defects due to FAI. This study suggests that AMIC is a valid procedure to repair medium-sized chondral defects on the acetabular side of the hip found during treatment of FAl and lead to long-term favorable outcomes. AMIC, due to its minimal invasiveness, single-stage procedure and proven safety, may be considered as a first choice treatment with respect to minimizing therapy time and costs.

\section{References}

1. Sussman PS, Ranawatt AS, Lipman J, et al. Arthroscopic versus open osteoplasty of the head-neck junction: a cadaveric investigation. Arthroscopy. 2007;23-12:1257-64.

2. McCarthy JC, Lee J. Hip arthroscopy. Indications and technical pearls. Clin Orthop Rel Res. 2005;441:180-7.

3. Tibor LM, Sekiya JK. Differential diagnosis of pain around the hip joint. Current concepts. Arthroscopy. 2008;24-12:1407-21

4. Ganz R, Parvizi J, Beck M, et al. Femoroacetabular impingement: a cause for osteoarthritis of the hip. Clin Orthop Rel Res. 2003;417:112-20.
5. Beck M, Kalhor M, Leunig M, et al. Hip morphology influences the pattern of damage to the acetabular cartilage: femoroacetabular impingement as a cause of early osteoarthritis of the hip. J Bone Joint Surg Br. 2005;87-B:1012-1018.

6. Cole BJ, Gomoll AH. Biologic joint reconstruction. Alternatives to arthroplasty. 2009 Ed Slack Inc. NJ, USA.

7. Lienert JJ, Rodkey WG, Steadman JR, Philippon MJ, Sekiya JK Microfracture techniques in hip arthroscopy. Oper Tech Orthop. 2005;15:267-272 .

8. McGill KC, Bush-Joseph CA, Nho SJ. Hip microfracture: indications, technique, and outcomes. Cartilage. 2010;1:127-136.

9. Crawford K, Philippon MJ, Sekiya JK, Rodkey WG, Steadman JR. Microfracture of the hip in athletes. Clin Sports Med. 2006;25:327- 335.

10. Brittberg M, Lindahl A, Nilsson A, Ohlsson C, Isaksson O, Peterson $\mathrm{L}$. Treatment of deep cartilage defects in the knee with autologous chondrocyte transplantation. N Engl J Med. 1994; 331:889-895.

11. Philippon MJ, Schenker ML, Briggs KK, Maxwell RB. Can microfracture produce repair tissue in acetabular chondral defects? Arthroscopy. 2008;24:46-50.

12. Leunig M, Tibor L, Naal F, Ganz R, Steinwachs M. Surgical technique: second-generation bone marrow stimulation via surgical dislocation to treat hip cartilage lesions. Clin Orthop Relat Res. 2012;470: 3421-3431.

13. Outerbridge RE. The etiology of chondromalacia patellae. J Bone Joint Surg Br. 1961;43-B:752-57.

14. Fontana A, Bistolfi A, Crova M, Rosso F, Massazza G. Arthroscopic treatment of hip chondral defects: autologous chondrocyte transplantation versus simple debridement-a pilot study. Arthroscopy. 2012;28:322-329.

15. Fontana A. A novel technique for treating cartilage defects in the hip: a fully arthroscopic approach to using autologous matrix induced chondrogenesis. Arthrosc Tech. 2012;1: e63-e68.

16. Söderman $P$, Malchau $H$. Is the Harris hip score system useful to study the outcome of total hip replacement? Clin Orthop Relat Res. 2001;384:189-197.

17. Bardakos NV, Vasconcelos JC, Villar RN. Early outcome of hip arthroscopy for femoroacetabular impingement: the role of a femoral osteoplasty in symptomatic improvement. J Bone Joint Surg Br. 2008;90:1570-1575.

18. Karthikeyan S, Roberts S, Griffin D. Microfracture for acetabular chondral defects in patients with femoro acetabular impingement: results at second-look arthroscopic surgery. Am J Sports Med. 2012;40:2725-2730.

19. Haviv B, Singh PJ, Takla A, O'Donnell J. Arthroscopic femoral osteochondroplasty for cam lesions with isolated acetabular chondral damage. J Bone Joint Surg Br. 2010;92:629-633.

20. Horisberger M, Brunner A, Herzog RF. Arthroscopic treatment of femoral acetabular impingement in patients with preoperative generalized degenerative changes. Arthroscopy. 2010; 26:623-629.

21. Singh PJ, O'Donnell JM. The outcome of hip arthroscopy in Australian football league players: a review of 27 hips. Arthroscopy. 2010;26:743-749.

22. Byrd JW, Jones KS. Osteoarthritis caused by an inverted acetabular labrum: radiographic diagnosis and arthroscopic treatment. Arthroscopy. 2002;18:741-747.

23. Akimau P, Bhosale A, Harrison PE, et al. Autologous chondrocyte implantation with bone grafting for osteochondral defect due to posttraumatic osteonecrosis of the hip-a case report. Acta Orthop. 2006;77:333-336.

24. Fontana A, de Girolamo L. Sustained five-year benefit of autologous matrix-induced chondrogenesis for femoral acetabular impingement-induced chondral lesions compared with microfracture treatment Bone Joint J. 2015;97-B, No.5, MAY 2015. 
25. Murphy CM, Matsiko A, Haugh MG, Gleeson JP, O'Brien FJ. Mesenchymal stem cell fate is regulated by the composition and mechanical properties of collagen-glycosaminoglycan scaffolds. J Mech Behav Biomed Mater. 2012;11:53-62.

26. Fuss M, Ehlers EM, Russlies M, Rohwedel J, Behrens P. Characteristics of human chondrocytes, osteoblasts and fibroblasts seeded onto a type I/III collagen sponge under different culture conditions. A light, scanning and transmission electron microscopy study. Ann Anat. 2000;182:303-310 .

27. Gille J, Meisner U, Ehlers EM, Müller A, Russlies M, Behrens P. Migration pattern, morphology and viability of cells suspended in or sealed with fibrin glue: a histomorphologic study. Tissue Cell. 2005;37:339-348.

28. Benthien JP, Behrens P. Autologous matrix-induced chondrogenesis (AMIC): combining microfracturing and a collagen I/II matrix for articular cartilage resurfacing. Cartilage. 2010;1:6568.

29. Gille J, Schuseil E, Wimmer J, Gellissen J, Schulz AP, Behrens P. Mid-term results of autologous matrix-induced chondrogenesis for treatment of focal cartilage defects in the knee. Knee Surg Sports Traumatol Arthrosc. 2010;18:1456-1464.
30. Gille J, Behrens P, Volpi P et al. Outcome of autologous matrix induced chondrogenesis (AMIC) in cartilage knee surgery: data of the AMIC Registry. Arch Orthop Trauma Surg 2013;133: 87-93.

31. Kusano T, Jakob RP, Gautier E, Magnussen RA, Hoogewoud $\mathrm{H}$, Jacobi M. Treatment of isolated chondral and osteochondral defects in the knee by autologous matrix-induced chondrogenesis (AMIC). Knee Surg Sports Traumatol Arthrosc 2012;20:2109-2115.

32. Walther M, Martin K. Scaffold based reconstruction of focal full thickness talar cartilage defects. Clin Res Foot Ankle. 2013;1:115.

33. Wiewiorski M, Miska M, Kretzschmar M, Studler U, Bieri O, Valderrabano V. Delayed gadolinium-enhanced MRI of cartilage of the ankle joint: results after autologous matrix-induced chondrogenesis (AMIC)-aided reconstruction of osteochondral lesions of the talus. Clin Radiol. 2013;68:1031-1038.

34. Padulo J, Oliva F, Frizziero A, Maffulli N. Muscles, Ligaments and Tendons Journal. Basic principles and recommendations in clinical and field science research: 2016 Update. MLTJ. 2016;6(1):1-5. 